\title{
Specific Heat and the Ground State of $\mathrm{NiO}$
}

\author{
R.J. RADWANSKI ${ }^{a, b}$ AND Z. ROPKA ${ }^{a}$ \\ ${ }^{a}$ Center of Solid State Physics, Św. Filip 5, 31-150 Kraków, Poland \\ ${ }^{b}$ Institute of Physics, Pedagogical University, 30-084 Kraków, Poland \\ Working in the strongly-correlated crystal-field approach and in the \\ strong hybridization limit we calculated the temperature dependence of the \\ heat capacity of $\mathrm{NiO}$ in a wide temperature range, from zero temperature \\ to $1200 \mathrm{~K}$. Our calculations reproduce reasonably well experimental depen- \\ dence including the $\lambda$-type peak at $T_{\mathrm{N}}$ of $525 \mathrm{~K}$.
}

PACS numbers: 71.10.--w, 75.10.-b, 75.10.Dg

\section{Introduction}

The insulating ground state and magnetism of $\mathrm{NiO}$ and other $3 d$ monooxides is a subject of a fundamental controversy by more than 70 years [1-8]. Despite of its simplicity (two atoms, $\mathrm{NaCl}$ structure, well-defined antiferromagnetism (AF) with $T_{\mathrm{N}}$ of $525 \mathrm{~K}$ ) and enormous theoretical and experimental works the consistent description of its properties, reconciling its insulating state with the unfilled $3 d$ band is still not reached. Recently we have solved this problem [9]. We have found consistent description of the magnetism and the low-energy electronic structure of $\mathrm{NiO}$ consistent with other monooxides $\mathrm{FeO}$ and $\mathrm{CoO}$ within the strongly-correlated crystal-field approach [10-12] which is a part of the quantum atomistic solid state theory (QUASST).

The aim of this paper is to report the calculations of the temperature dependence of the heat capacity of $\mathrm{NiO}$ in a wide temperature range, from zero temperature to $1200 \mathrm{~K}$, passing the magnetic-ordering temperature.

\section{Theoretical outline}

In QUASST the insulating ground state of $\mathrm{NiO}$ results from the ionic state $\mathrm{Ni}^{2+} \mathrm{O}^{2-}$. These ionic states are formed during the formation of the compound. One can also say that such charge redistribution results from strong hybridization of $3 d(\mathrm{Ni})-2 p(\mathrm{O})$ states thanks which the nickel atoms are ready to give up two electrons and the oxygen atoms energetically prefer to accept these two electrons, becoming $\mathrm{O}^{2-}$ ions. The $\mathrm{O}^{2-}$ ions assume the full $2 p^{6}$ configuration and 
are magnetically and energetically silent. The $\mathrm{Ni}^{2+}$ ion has 8 electrons in the incomplete $3 d$ shell. We treat these 8 outer electrons of the $\mathrm{Ni}^{2+}$ ion as forming the highly-correlated electron system $3 d^{8}$ resembling the atomic/ionic situation (it justifies the used name with a word "atomistic"). The ground term of the strongly-correlated electron system $3 d^{8}\left(\mathrm{Ni}^{2+}\right.$ ion) is described by two Hund's rules yielding $S=1$ and $L=3$, i.e. the ground term ${ }^{3} F$. Such the localized strongly-correlated electron system interacts in a solid self-consistently with the charge and spin surroundings. The charge surrounding has the predominantly octahedral symmetry owing to the NaCl-type of structure of $\mathrm{NiO}$.

Our Hamiltonian for $\mathrm{NiO}$ consists of two terms: the single-ion-like term $H_{d}$ of the $3 d^{8}$ system and the $d-d$ intersite spin-dependent term. Calculations somehow resemble those performed for rare-earth systems, see e.g. Refs. [13, 14] and we apply this approach successfully to $3 d$ compounds $[12,15,16]$.

\section{Results and discussion}

The resulting electronic structure has been shown in Refs. [9, 17]. Here we resume that the single-ion calculations, with $\lambda_{\mathrm{s}-\mathrm{o}}=-41 \mathrm{meV}$ and the octupolar crystalline electric field (CEF) parameter $B_{4}=+21 \mathrm{~K}(2 \mathrm{meV})$ yield the cubic 3 -fold degenerated subterm ${ }^{3} A_{2 \mathrm{~g}}$ as the ground state with higher group of localized states at 1.2 and $2.1 \mathrm{eV}[9,17]$. The sign of $B_{4}$ is directly related to the oxygen anion surroundings. We have calculated its value in Refs. [9, 17] from first principles taking only $\left\langle r_{d}^{4}\right\rangle$ to be $10.5 a_{\mathrm{B}}^{4}$.

In Fig. 1 we recall Fig. 2 of Ref. [17] where the splitting of the lowest cubic subterm ${ }^{3} A_{2 \mathrm{~g}}$ is shown - the cubic subterm ${ }^{3} A_{2 \mathrm{~g}}$ is the most important for low- and room-temperature properties because the higher states are practically not thermally populated below, say, $1000 \mathrm{~K}$. From this figure it is seen that the triplet states of ${ }^{3} A_{2 \mathrm{~g}}$ are split in the magnetically-ordered state, i.e. below $525 \mathrm{~K}$.

In Fig. 2 we present the calculated temperature dependence of the $3 d$-electron contribution to the heat capacity of $\mathrm{NiO}$. One can see that together with the lattice heat, approximated by the Debye temperature of $650 \mathrm{~K}$, our calculations nicely reproduce the experimental variation in the whole wide temperature range including the lambda-type peak at $T_{\mathrm{N}}$. The spike-like peak at $T_{\mathrm{N}}$ is in good agreement with experimental data [18] obtained on a single-crystal specimen that yields "very large, very narrow peak of $65 \mathrm{cal} /(\mathrm{K} \mathrm{mol})$ " [19]. Moreover, we have got that the magnetically-ordered state of $\mathrm{NiO}$ has lower energy than the paramagnetic one by $3.4 \mathrm{~kJ} / \mathrm{mol}$ (=35 meV/ion) at $0 \mathrm{~K}$. Of course, the energies of magnetic and paramagnetic states become equal at $T_{\mathrm{N}}$. We could fit the experimental temperature dependence of the heat capacity better by allowing, for instance, for the temperature dependence of the Debye temperature $\Theta_{\mathrm{D}}$ of $650 \mathrm{~K}$ or the existence of short-range magnetic correlations. Although it is reasonable to think that $\Theta_{\mathrm{D}}(T)$ is not constant in so wide temperature range we did not make such calculations because it would be a mere speculation. We are quite satisfied with 


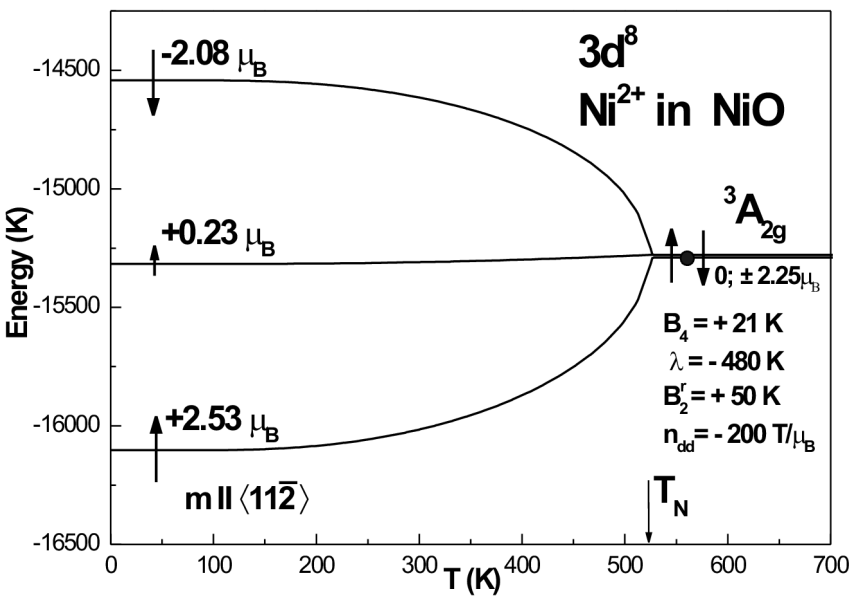

Fig. 1. The calculated temperature dependence of the energy of the three lowest levels originating from ${ }^{3} A_{2 \mathrm{~g}}$ cubic subterm showing the splitting of the triplet in the magnetic state (below $T_{\mathrm{N}}=525 \mathrm{~K}$ ). It presents the low-energy electronic structure of $\mathrm{NiO}$, below $200 \mathrm{meV}$.

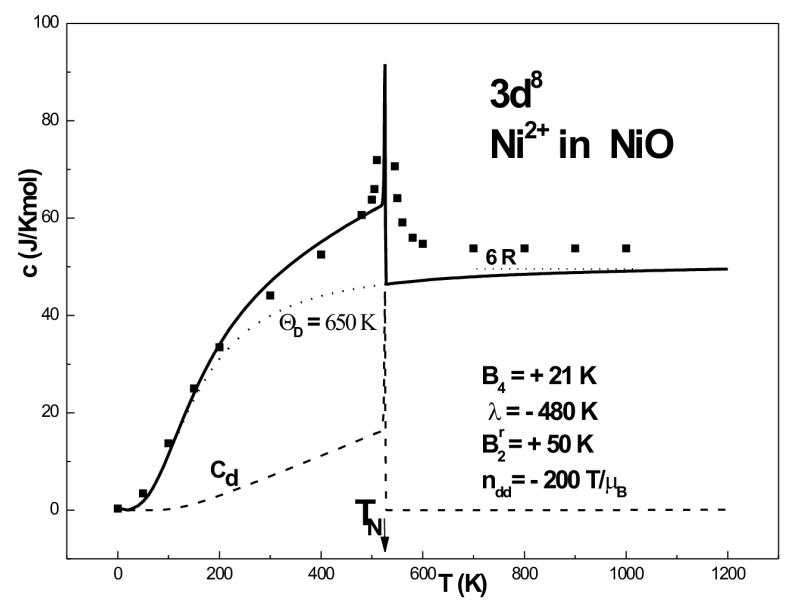

Fig. 2. The calculated temperature dependence of the $3 d$ contribution to the heat capacity of $\mathrm{NiO}$ with $\Theta_{\mathrm{D}}$ of $650 \mathrm{~K}$. The spike peaks to $214 \mathrm{~J} /(\mathrm{K} \mathrm{mol})$. This calculated $c_{d}(T)$ dependence is in nice agreement with experimental data shown in Refs. [18] and [19], where the spike reaches a giant value of $272 \mathrm{~J} /(\mathrm{K} \mathrm{mol})$.

the obtained agreement because we are interested in the overall wide-temperature description, both in the antiferromagnetic and paramagnetic state. To our best knowledge we do not know any paper and any theoretical approach which could in one consistent way describe both the ground state and thermodynamics, with the magnetic-paramagnetic transition. 
We would like to point out that our approach should not be considered as the treatment of an isolated ion only — we consider the $\mathrm{Ni}^{2+}$ ion in the oxygen octahedron. The physical relevance of our calculations to macroscopic $\mathrm{NiO}$ is obvious - the $\mathrm{NaCl}$ structure is built up from the edge sharing $\mathrm{Ni}^{2+}$-centered octahedra.

\section{Summary}

We have calculated the temperature dependence of the heat capacity of $\mathrm{NiO}$ in a wide temperature range, from zero temperature to $1200 \mathrm{~K}$. Our calculations reproduce reasonably well the overall experimental dependence including the $\lambda$-type peak at $T_{\mathrm{N}}$ of $525 \mathrm{~K}$. We are fully aware that a final description of the heat capacity of $\mathrm{NiO}$ has to take into account many other, but relatively small, contributions and has to go beyond the single-site, mean-field approach but, we believe, it should start from such atomic-based electronic structure.

In our approach eight $d$ electrons, of all 10 electrons of the nickel atom, are fully localized and strongly correlated. We take into account very strong electron correlations, the strong $3 d-2 p$ hybridization and spin-orbit interactions. Our theoretical approach is consistent with experimental revealing of the orbital magnetic moment in $\mathrm{NiO}$ [20]. In our approach $\mathrm{NiO}$ is insulator both in the antiferromagnetic state and in the paramagnetic state.

\section{References}

[1] T.M. Schuler, D.L. Ederer, S. Itza-Ortiz, G.T. Woods, T.A. Callcott, J.C. Woicik, Phys. Rev. B 71, 115113 (2005).

[2] L.-C. Duda, T. Schmitt, M. Magnuson, J. Forsberg, A. Olsson, J. Nordgren, K. Okada, A. Kotani, Phys. Rev. Lett. 96, 067402 (2006).

[3] A.B. Shick, A.I. Liechtenstein, W.E. Pickett, Phys. Rev. B 60, 10763 (1999).

[4] K. Terakura, A.R. Williams, T. Oguchi, J. Kubler, Phys. Rev. Lett. 52, 1830 (1984).

[5] S.V. Faleev, M. van Schilfgaarde, T. Kotani, Phys. Rev. Lett. 93, 126406 (2004).

[6] V.I. Anisimov, F. Aryasetiawan, A.I. Liechtenstein, J. Phys., Condens. Matter 9, 767 (1997).

[7] O. Bengone, M. Alouani, P. Blochl, J. Hugel, Phys. Rev. B 62, 16392 (2000).

[8] D.I. Khomskii, G.A. Sawatzky, Solid State Commun. 102, 87 (1997).

[9] R.J. Radwanski, Z. Ropka, Acta Physica 1, 26 (2006).

[10] R.J. Radwanski, Z. Ropka, Acta Phys. Pol. A 97, 963 (2000); arXiv/condmat/0005471 (2000).

[11] R.J. Radwanski, Z. Ropka, in: New Developments in Field Theory, Ed. O. Kovras, Nova Science Publ., New York 2006, p. 93; arXiv/cond-mat/0404713 (2004).

[12] R.J. Radwanski, Z. Ropka, Physica B 345, 107 (2004); arXiv/cond-mat/0306695 (2003). 
[13] R.J. Radwanski, N.H. Kim-Ngan, F.E. Kayzel, J.J.M. Franse, D. Gignoux, D. Schmitt, F.Y. Zhang, J. Phys., Condens. Matter 4, 8853 (1992).

[14] R.J. Radwanski, J. Phys., Condens. Matter 8, 10467 (1996).

[15] Z. Ropka, R. Michalski, R.J. Radwanski, Phys. Rev. B 63, 172404 (2001).

[16] Z. Ropka, R.J. Radwanski, Phys. Rev. B 67, 172401 (2003).

[17] R.J. Radwanski, Z. Ropka, Acta Physica 5, 17 (2007).

[18] V.P. Zhuze, O.N. Novruzov, A.I. Shelykh, Fiz. Tverd. Tela 11, 1287 (1969); Sov. Phys. Solid State 11, 1044 (1970).

[19] J.E. Keem, J.M. Honig, Selected Electrical and Thermal Data of Undoped Nickel Oxide, Center for Information and Numerical Data, Cindas Report 52 (August 1978), Purdue University.

[20] W. Neubeck, C. Vettier, F. de Bergevin, F. Yakhou, D. Mannix, O. Bengone, M. Alouani, A. Barbier, Phys. Rev. B 63, 134430 (2001). 